\title{
Expression of EpCam and villin in Barrett's esophagus and in gastric cardia
}

\author{
Mario Anders ${ }^{\mathrm{a}, *}$, Mario Sarbia ${ }^{\mathrm{b}}$, Carsten Grotzinger $^{\mathrm{a}}$, Alexander Meining ${ }^{\mathrm{c}}$, Heinz Hofler ${ }^{\mathrm{d}}$, \\ Bertram Wiedenmann ${ }^{\mathrm{a}}$ and Thomas Rosch ${ }^{\mathrm{a}}$ \\ ${ }^{a}$ Department of Internal Medicine, Divisions of Gastroenterology and Hepatology, Charité Medical School- \\ Campus Virchow, Berlin, Germany \\ ${ }^{\mathrm{b}}$ Department of Pathology, Unfallkrankenhaus Berlin/Sana Klinikum Lichtenberg, Berlin, Germany \\ ${ }^{\mathrm{c}}$ Department of Internal Medicine II, Technical University of Munich, Munich, Germany \\ ${ }^{\mathrm{d}}$ Institute of Pathology, Technical University of Munich, Munich, Germany
}

\begin{abstract}
In the current study we aimed to clarify the potential of EpCAM and villin as in vivo biomarkers for both Barrett esophagus (BE)-associated neoplasia and BE versus cardiac mucosa. Immunohistochemical staining in $\mathrm{BE}$ with various degrees of intraepithelial neoplasia (IN), Barrett carcinoma (BC) and in normal cardiac mucosa (CM) revealed a lack of EpCam and villin in squamous esophageal epithelium. All specimens of IN and BC showed EpCam with varying staining intensities. In 57\% of CM samples a weak signal was detected; the remainder displayed strong EpCam expression. Villin was found in $97 \%$ of BE specimens and in all those with IN; $37 \%$ of BC and $75 \%$ of CM specimens were also positive. We conclude that expression of EpCam and villin differs only between squamous epithelium and BE. Determination of these proteins does not allow discrimination between different degrees of neoplasia or between esophageal intestinal metaplasia and cardiac mucosa.
\end{abstract}

Keywords: Barrett, metaplasia, dysplasia, neoplasia, villin, EpCam, endoscopy, biomarker

\section{Introduction}

Barrett's esophagus (BE) represents the acquired replacement of squamous epithelium lining the distal esophagus by columnar cells containing goblet cells, also called "specialized intestinal metaplasia". BE is recognized as a precancerous condition requiring regular surveillance endoscopies with biopsies [1]. The frequency of BE-associated adenocarcinoma has consistently increased over recent decades [2,3]. Carcinoma in BE develops via dysplasia, also termed intraepithelial neoplasia, which is characterized by architectural abnormalities (glandular distortion and crowding, papillary extensions, and villiform configuration

*Corresponding author: Charité - Universitätsmedizin Berlin, Campus Virchow - Klinikum, Augustenburger Platz 1, 13353 Berlin, Germany. Tel.: +49 30450 553313; Fax: +49 30450 553902; E-mail: mario.anders@charite.de. of the mucosa surface) and changes in cytological measures (variation in nuclear size and shape, increased nuclear-to-cytoplasm ratio, hyperchromatism, and increased number of abnormal mitoses) [4].

The diagnostic work-up of BE presents two challenges in clinical practice:

1. The diagnosis of BE rests on endoscopy plus histologic confirmation, but among patients with short or very short forms it may be difficult to identify which patients should be biopsied. This is because endoscopy may be quite unreliable in defining a short BE segment. The precise recognition of the gastro-esophageal transition is of crucial impact as it defines whether intestinal metaplasia belongs to the esophagus, representing a short segment BE [5] as opposed to proximal gastric metaplasia. The risk of progression to Barrett carcinoma (BC) from intraepithelial neoplastic lesions in short segment BE seems to be 
equivalent to the risk with longer Barrett's epithelium [6]. Thus, a marker for Barrett epithelium versus proximal gastric (cardiac) mucosa would be very helpful.

2. Once BE is established, surveillance consists of endoscopic inspection with biopsy of all suspicious focal areas as well as stepwise fourquadrant biopsies. Such an approach is commonly felt to be time-consuming and, due to an inherent sampling error, only partially effective. The use of markers for imaging, employing antibodies to highlight areas of neoplasia which are endoscopically invisible, would help in performing targeted biopsies only, thus saving time.

To achieve this purpose several techniques, such as tissue fluorescence after administration of various substances (photodynamic diagnosis) and tissue auto fluorescence endoscopy, have been evaluated but have not yielded consistent results [7]. Previously in vivo application of biomarkers during endoscopy has been demonstrated, using a fluorescence-labeled antibody against carcinoembryonic antigen for the detection of colonic adenoma and carcinoma [8], but no data regarding in vivo application of biomarkers for $\mathrm{BE}$ have yet been published. However, based on findings in human tissue samples, EpCam and villin have been proposed as sensitive and specific indicators of BE [9-13].

EpCAM, a 40-kDa transmembrane glycoprotein, is expressed in various human epithelia including bowel mucosa, but not in the gastric mucosa or squamous epithelium of the esophagus [13,14]. Barrett's metaplasia however strongly expresses this protein [13]. Villin is a $\mathrm{Ca}^{2+}$-dependent actin-binding protein, necessary for the constitution of apical microvilli in the intestinal epithelium [15], and can be found in the small bowel, but not in the normal gastric mucosa $[9,10]$. While high expression of villin has been detected in metaplastic BE, squamous esophagus is considered to be villin-negative $[9,10,12]$.

In the current study we have investigated EpCam and villin expression in the diverse stages of $\mathrm{BE}$ and in the gastric cardia region. Thereby, we aimed to evaluate the potential utility of these proteins as in vivo biomarkers in order to improve endoscopic detection and surveillance in individuals with BE.

\section{Material and methods}

\subsection{Study population and tissues}

Biopsy tissue specimens were obtained from $10 \mathrm{pa}-$ tients (five men; mean age 60 years, range 36-82) with previously diagnosed BE who had undergone endoscopic surveillance according to standards during 2002 at the Department of Internal Medicine II, Technical University of Munich, Munich, Germany. Written informed consent for immunohistochemical evaluation of the samples was obtained from all patients. The procedure included 4 quadrant biopsies starting at the proximal cardia folds, every $2 \mathrm{~cm}$ up to the proximal BE margin, which in most cases contained adjacent squamous epithelium. This normal squamous epithelium from the most proximal biopsies was taken as controls and was present in at least one biopsy from each patient. The specimens included: BE, that was without neoplasia ( $n=66$ ), indefinite for intraepithelial neoplasia (IDIN) $(n=8)$, with low grade intraepithelial neoplasia (LGIN) $(n=10)$, and with high grade intraepithelial neoplasia (HGIN) $(n=2)$; Barrett's carcinoma (BC) $(n=16)$; and cardiac mucosa (CM) $(n=12)$.

Histological categorization of tissue types was performed according to standard criteria by two senior pathologists with a special interest in gastrointestinal pathology (Mario Sarbia, Ingrid Becker) on the basis of the H\&E-stained slides. Cardiac mucosa was characterized by loosely packed glands composed only of mucous epithelia. The surface was covered by gastric foveolar epithelium [16]. Metaplastic Barrett's mucosa was characterized by incomplete type of intestinal metaplasia with a mixture of goblet cells and columnar cells that have cytological features of gastric foveolar cells or intestinal absorptive cells (usually without brush border) [17]. Barrett mucosa indefinite for intraepithelial neoplasia, low grade intraepithelial neoplasia and high grade intraepithelial neoplasia were defined histologically according to the criteria of Montgomery et al. [18]. Barrett's carcinoma was defined as an adenocarcinoma with concomitant histological verification of metaplastic Barrett's mucosa and/or intraepithelial neoplasia in Barrett's mucosa. Intramucosal carcinoma was defined by either penetration of neoplastic cells through the basement membrane and/or the presence of architecturally complex collections of neoplastic cells in the lamina propria that could not be explained by the presence of pre-existing Barrett's mucosa [19].

\subsection{Construction of tissue microarrays and immunohistological investigations}

Tissue microarrays were constructed as described previously [20] using a tissue-arraying instrument (Beecher Instruments, Silver Spring, MD, USA). For 
the subsequent immunohistochemical study, $4 \mu \mathrm{m}$ sections from the tissue microarrays were mounted on Superfrost-plus slides. Subsequently, sections were stained according to standard procedures using monoclonal antibodies against EpCam (clone AUA1, dilution 1:200; Abcam, Cambridge, UK) and villin (clone ID2C3, dilution 1:100; Immunotech, Marseille, France). Each run included appropriate positive controls. Negative controls were treated identically to the tumor samples including the antigen retrieval procedure, except that the primary antibody was replaced by mouse non-immune serum. In negative controls no signals were detectable.

Expression of EpCam and villin, respectively, was considered positive if at least $10 \%$ of the cell type of interest in at least one of the three tissue cores showed staining comparable to positive controls.

\section{Results}

To determine the expression of EpCam and villin in normal esophageal squamous epithelium, samples were stained immunohistochemically. Neither of the two proteins displayed immunopositivity in stratified squamous epithelium.

In non-neoplastic Barrett's metaplasia, EpCam expression was present in all samples: a signal of strong intensity was found in $87 \%$ of the specimens, while $13 \%$ displayed a weak signal. Samples of IDIN were found to be EpCam positive in $85 \%$ of the cases. Furthermore, all specimens with intraepithelial neoplastic lesions were found to be positive for EpCam expression. Staining intensities differed according to the degree of intraepithelial neoplasia: those classified as LGIN revealed a strong, specific signal in $100 \%$ of the cases, while specimens with HGIN only showed a weak signal. All Barrett carcinoma specimens were EpCampositive; however staining intensities varied considerably, with $66 \%$ presenting a weak and $34 \%$ a strong signal. In $57 \%$ of samples of cardiac mucosa a weak signal was detected, while the remaining cases displayed strong EpCam expression (Table 1; Fig. 1).

Villin was found in $97 \%$ of Barrett's metaplasia samples and in all IDIN, LGIN, and HGIN specimens, while only $37 \%$ of the Barrett carcinoma samples revealed villin expression. Of the cardiac mucosa samples, $75 \%$ displayed villin immunopositivity (Table 1 ; Fig. 1).

\section{Discussion}

The objective of this study was to investigate the expression of EpCam and villin in the various stages of BE (non-neoplastic and neoplastic) and in the gastric cardia. The clinical background to this investigation was the potential utility of these proteins as in vivo biomarkers to improve the endoscopic assessment of $\mathrm{BE}$, regarding both diagnosis of $\mathrm{BE}$ and recognition of neoplasia in established BE. Our results support the concept that EpCAM and villin are not expressed in stratified esophageal epithelium, as suggested in previous studies investigating mRNA and protein expression of these molecules [9-13]. Our findings also reveal abundant expression of EpCam and villin in BE, in agreement with previous investigations [9-13]; however, discrimination of a) intestinal metaplasia, distinguishing BE from proximal gastric mucosa, and b) discrimination between non-neoplastic and neoplastic BE was not reliably possible using these markers.

In comparison with previous reports, the current study is the first detailed assessment of EpCam expression in the different stages of BE. However, even though EpCam staining intensity in LGIN was different from that seen in HGIN, the use of EpCam as biomarker for the degree of intraepithelial neoplasia cannot be justified since no clear-cut distinction (positive vs. negative) could be found. The same is true for villin, consistent with preliminary data from previous studies that analyzed a lower number of neoplasia-positive biopsy sites: Ouatu-Lascar et al. could not find any relation between villin protein expression and Barrett dysplasia in 14 patients, including one with HGIN [11]. Regalado et al. analyzed villin expression in Barrett's metaplasia ( $n=21$ ), including 12 cases with intraepithelial neoplasia, and found all biopsies containing the various entities to be highly villin-positive. Unfortunately, no information on further classification regarding LGIN or HGIN is given [12]. Kumble et al. detected no correlation between villin expression in BE $(n=6)$ and presence of intraepithelial neoplasia. However, no patient in this study displayed HGIN [9].

As we frequently observed weak or even no expression of EpCam and villin in Barrett carcinoma, as well as their expression in proximal gastric mucosa, we conclude that use of these proteins as biomarker for Barrett carcinoma is not appropriate. Kumble et al. also detected either no or very low villin expression with immunoblotting in four patients with Barrett carcinoma [9]. Nevertheless, our data contrast with those of other studies: Martin et al. found moderate to strong 
Table 1

Expression of EpCam and villin as determined by immunohistochemistry

\begin{tabular}{lcccrr}
\hline & $\begin{array}{c}\text { EpCam } \\
\mathrm{n}=\end{array}$ & weak positive & strong positive & $\begin{array}{c}\text { Villin } \\
\mathrm{n}=\end{array}$ & positive \\
\hline Barrett metaplasia & 60 & 8 & 52 & 66 & 64 \\
Indefinite for IN & 7 & 1 & 6 & 8 & 8 \\
Low grade IN & 6 & 0 & 6 & 10 & 10 \\
High grade IN & 2 & 2 & 0 & 2 & 2 \\
Barrett carcinoma & 12 & 8 & 4 & 16 & 6 \\
Cardiac mucosa & 7 & 4 & 3 & 12 & 9 \\
\hline
\end{tabular}

The variation in the numbers of tissue samples assessable for EpCam and villin immunostaining was due to technical reasons, e.g. loss of tissue cores during staining procedures. "IN": intraepithelial neoplasia.
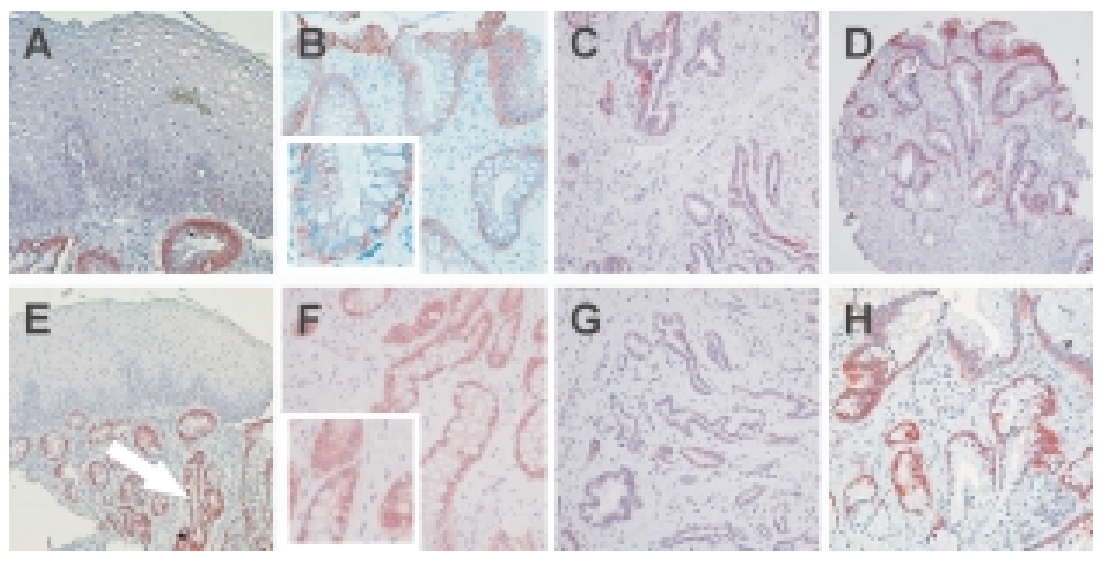

Fig. 1. Esophageal squamous epithelium lacks expression of EpCAM (A) and villin (E) (arrow marks immuno-positive underlying Barrett's metaplasia). Expression of EpCAM (B) and villin (F) in metaplastic Barrett's mucosa. Representative areas in higher magnification display the subcellular distribution of EpCAM predominantly at the plasma membrane and villin showing diffuse intracellular localization (additional panels in B, F). Reduced expression of EpCAM (C) and villin (G) in a Barrett's adenocarcinoma characterized by architecturally complex glands in a desmoplastic stroma. In cardiac mucosa expression of both proteins EpCAM (D) and villin (H) is evident. (Magnification: A-C, F-H: $\times 200$; D,E: $\times 100)$.

EpCam protein expression in 18 carcinomas of the gastroesophageal junction and the upper stomach [21]. Furthermore, a high level of EpCam expression in Barrett carcinoma specimens was observed by Kumble et al. [9]. Regalado et al. [12] detected high villin expression in Barrett carcinoma $(n=30)$ and gastric cardia adenocarcinomas $(n=16)$; based on these findings the authors suggested that determination of villin serum levels might be useful in monitoring patients who had undergone esophagectomy for adenocarcinoma. The difference between these findings and those of the current study may be accounted for by the use of different methods (immunoblotting, immunohistochemistry, southern and northern blotting), various staining procedures, and diverse antibodies.

Our results also show that there is no great difference between BE and gastric mucosa regarding EpCam and villin expression. Therefore, it must be concluded that neither protein could serve as an appropriate biomark- er to discriminate BE from gastric mucosa, confirming preliminary reports by Spurr et al. [22] and Martin and colleagues [21]. Interestingly, our data regarding villin expression differ from those of previous studies: samples of gastric mucosa have been found to be villinnegative by Kumble et al. $(n=23)$ and MacLennan et al. $(n=5)[9,10]$. These discrepancies may be explained by differences in biopsy site, that is, the mucosa of the gastric cardia region in the current study, and the gastric fundus in Kumble et al.'s investigation; the location of the gastric mucosal biopsies studied by MacLennan et al. is unfortunately not stated. Moreover, both these groups employed different methods from ours (immunoblotting, northern blotting) that may explain the divergent results.

Recently, other proteins have been suggested to distinguish intestinal metaplasia of the esophagus from proximal gastric mucosa, such as cytokeratins, mucins, 45MI, and mAb Das-1. A BE-specific CK7/20 pat- 
tern, with CK20 detected in surface epithelial cells and in superficial but not deep glands, and CK7 detected in both superficial and deep glands, has been proposed $[23,24]$. However, the sensitivity and specificity of this CK7/20 pattern for BE has been disputed [2529]. The monoclonal antibody, mAb Das-1, that had been suggested to detect Barrett's epithelium with high sensitivity and specificity failed to distinguish between short-segment BE and intestinal metaplasia of the gastroesophageal junction [26,30]. Finally, using 45 M1, an antibody recognizing $\mathrm{M} 1$ gastric mucin antigen, no significant differences between intestinal metaplasia in the esophagus, the gastroesophageal junction, or the gastric antrum were detected [26].

In conclusion our findings in a limited population size again show that no biomarker has yet emerged that will reliably permit identification of intraepithelial neoplasia [31] or distinguish intestinal metaplasia in BE from cardiac epithelium [32]. Thus, the search continues for biomarkers that can be used in the endoscopic surveillance of BE.

\section{Acknowledgements}

We thank PD Dr. Ingrid Becker for cooperation in classification and grading of the tissue specimens.

\section{References}

[1] R.E. Sampliner, Practice guidelines on the diagnosis, surveillance, and therapy of Barrett's esophagus. The Practice Parameters Committee of the American College of Gastroenterology, Am J Gastroenterol 93 (1998), 1028-1032.

[2] W.J. Blot and J.K. McLaughlin, The changing epidemiology of esophageal cancer, Semin Oncol 26 (1999), 2-8.

[3] J. Powell and C.C. McConkey, The rising trend in oesophageal adenocarcinoma and gastric cardia, Eur J Cancer Prev 1 (1992), 265-269.

[4] J.F. Flejou, Barrett's oesophagus: from metaplasia to dysplasia and cancer, Gut 54(Suppl 1) (2005), i6-12.

[5] P. Sharma, T.G. Morales and R.E. Sampliner, Short segment Barrett's esophagus-the need for standardization of the definition and of endoscopic criteria, Am J Gastroenterol 93 (1998), 1033-1036.

[6] R.E. Rudolph, T.L. Vaughan, B.E. Storer, R.C. Haggitt, P.S. Rabinovitch, D.S. Levine and B.J. Reid, Effect of segment length on risk for neoplastic progression in patients with Barrett esophagus, Ann Intern Med 132 (2000), 612-620.

[7] M.A. Kara and J.J. Bergman, Autofluorescence imaging and narrow-band imaging for the detection of early neoplasia in patients with Barrett's esophagus, Endoscopy 38 (2006), 627631.
[8] R. Keller, G. Winde, H.J. Terpe, E.C. Foerster and W. Domschke, Fluorescence endoscopy using a fluorescein-labeled monoclonal antibody against carcinoembryonic antigen in patients with colorectal carcinoma and adenoma, Endoscopy 34 (2002), 801-807.

[9] S. Kumble, M.B. Omary, L.F. Fajardo and G. Triadafilopoulos, Multifocal heterogeneity in villin and Ep-CAM expression in Barrett's esophagus, Int J Cancer 66 (1996), 48-54.

[10] A.J. MacLennan, M.B. Orringer and D.G. Beer, Identification of intestinal-type Barrett's metaplasia by using the intestinespecific protein villin and esophageal brush cytology, Mol Carcinog 24 (1999), 137-143.

[11] R. Ouatu-Lascar, R.C. Fitzgerald and G. Triadafilopoulos, Differentiation and proliferation in Barrett's esophagus and the effects of acid suppression, Gastroenterology 117 (1999), 327-335.

[12] S.P. Regalado, Y. Nambu, M.D. Iannettoni, M.B. Orringer and D.G. Beer, Abundant expression of the intestinal protein villin in Barrett's metaplasia and esophageal adenocarcinomas, $\mathrm{Mol}$ Carcinog 22 (1998), 182-189.

[13] N.A. Wong, B.F. Warren, J. Piris, N. Maynard, R. Marshall and W.F. Bodmer, EpCAM and gpA33 are markers of Barrett's metaplasia, J Clin Pathol 59 (2006), 260-263.

[14] M. Balzar, M.J. Winter, C.J. de Boer and S.V. Litvinov, The biology of the 17-1A antigen (Ep-CAM), J Mol Med 77 (1999), 699-712.

[15] A. Bretscher and K. Weber, Villin: the major microfilamentassociated protein of the intestinal microvillus, Proc Natl Acad Sci USA 76 (1979), 2321-2325.

[16] D.A. Owen, Stomach, in Histology for Pathologists-3 ${ }^{r d}$ ed. Stacey E. Mills (ed)., Lippincott Williams \& Wilkins, Philadelphia 2007. In., 2007, pp. 589-602.

[17] R.C. Haggitt, Barrett's esophagus, dysplasia, and adenocarcinoma, Hum Pathol 25 (1994), 982-993.

[18] E. Montgomery, J.R. Goldblum, J.K. Greenson, M.M. Haber, L.W. Lamps, G.Y. Lauwers, A.J. Lazenby, D.N. Lewin, M.E. Robert, K. Washington, M.L. Zahurak and J. Hart, Dysplasia as a predictive marker for invasive carcinoma in Barrett esophagus: a follow-up study based on 138 cases from a diagnostic variability study, Hum Pathol 32 (2001), 379-388.

[19] A.H. Ormsby, R.E. Petras, W.H. Henricks, T.W. Rice, L.A. Rybicki, J.E. Richter and J.R. Goldblum, Observer variation in the diagnosis of superficial oesophageal adenocarcinoma, Gut 51 (2002), 671-676.

[20] B. H. von Rahden, H. J. Stein, F. Puhringer-Oppermann and M. Sarbia, c-myc amplification is frequent in esophageal adenocarcinoma and correlated with the upregulation of VEGF-A expression, Neoplasia 8 (2006), 702-707.

[21] I.G. Martin, S.G. Cutts, K. Birbeck, S. Gray and P. Quirke, Expression of the 17-1A antigen in gastric and gastrooesophageal junction adenocarcinomas: a potential immunotherapeutic target? J Clin Pathol 52 (1999), 701-704.

[22] N.K. Spurr, H. Durbin, D. Sheer, M. Parkar, L. Bobrow and W.F. Bodmer, Characterization and chromosomal assignment of a human cell surface antigen defined by the monoclonal antibody AUAI, Int J Cancer 38 (1986), 631-636.

[23] A. Couvelard, J.M. Cauvin, D. Goldfain, A. Rotenberg, M. Robaszkiewicz and J.F. Flejou, Cytokeratin immunoreactivity of intestinal metaplasia at normal oesophagogastric junction indicates its aetiology, Gut 49 (2001), 761-766.

[24] A.H. Ormsby, J.R. Goldblum, T.W. Rice, J.E. Richter, G.W. Falk, M.F. Vaezi and T.L. Gramlich, Cytokeratin subsets can reliably distinguish Barrett's esophagus from intestinal metaplasia of the stomach, Hum Pathol 30 (1999), 288-294. 
[25] H.M. El Zimaity and D.Y. Graham, Cytokeratin subsets for distinguishing Barrett's esophagus from intestinal metaplasia in the cardia using endoscopic biopsy specimens, Am J Gastroenterol 96 (2001), 1378-1382.

[26] J.N. Glickman, H. Wang, K.M. Das, R.K. Goyal, S.J. Spechler, D. Antonioli and R.D. Odze, Phenotype of Barrett's esophagus and intestinal metaplasia of the distal esophagus and gastroesophageal junction: an immunohistochemical study of cytokeratins 7 and 20, Das-1 and 45 MI, Am J Surg Pathol 25 (2001), 87-94.

[27] I. Jovanovic, M. Tzardi, I.A. Mouzas, M. Micev, P. Pesko, T. Milosavljevic, M. Zois, M. Sganzos, G. Delides and P. Kanavaros, Changing pattern of cytokeratin 7 and 20 expression from normal epithelium to intestinal metaplasia of the gastric mucosa and gastroesophageal junction, Histol Histopathol 17 (2002), 445-454.

[28] I.A. Mohammed, C.J. Streutker and R.H. Riddell, Utilization of cytokeratins 7 and 20 does not differentiate between Barrett's esophagus and gastric cardiac intestinal metaplasia, Mod Pathol 15 (2002), 611-616.

[29] M. Sarbia, A. Donner, C. Franke and H.E. Gabbert, Distinction between intestinal metaplasia in the cardia and in Barrett's esophagus: the role of histology and immunohistochemistry, Hum Pathol 35 (2004), 371-376.

[30] K.M. Das, I. Prasad, S. Garla and P.S. Amenta, Detection of a shared colon epithelial epitope on Barrett epithelium by a novel monoclonal antibody, Ann Intern Med 120 (1994), $753-756$.

[31] D.T. McManus, A. Olaru and S.J. Meltzer, Biomarkers of esophageal adenocarcinoma and Barrett's esophagus, Cancer Res 64 (2004), 1561-1569.

[32] C.P. Morales and S.J. Spechler, Intestinal metaplasia at the gastroesophageal junction: Barrett's, bacteria, and biomarkers, Am J Gastroenterol 98 (2003), 759-762. 


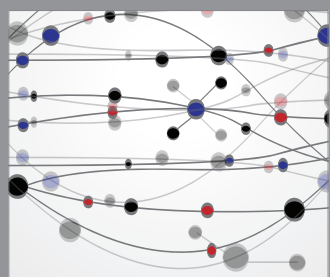

The Scientific World Journal
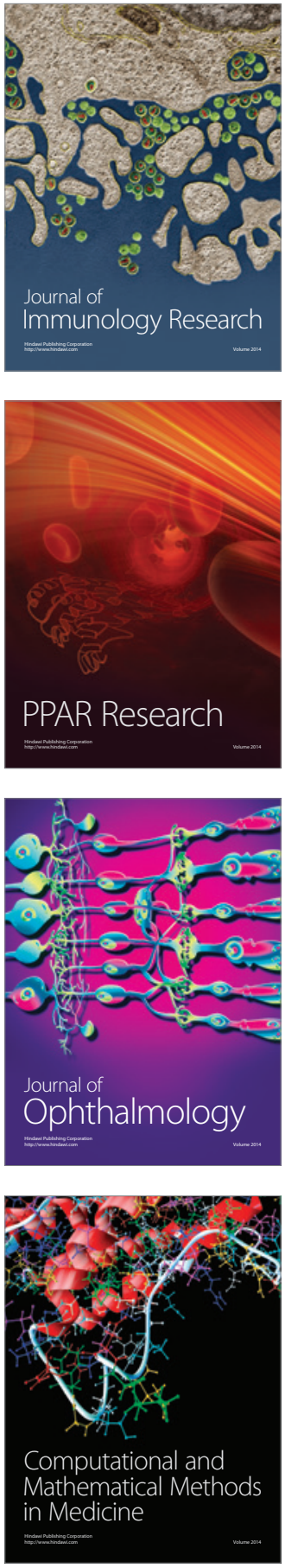

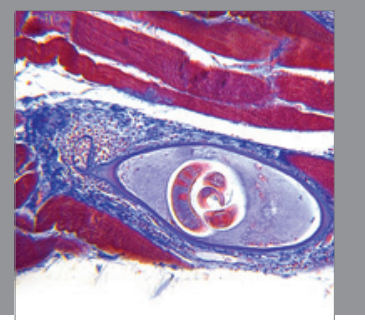

Gastroenterology

Research and Practice
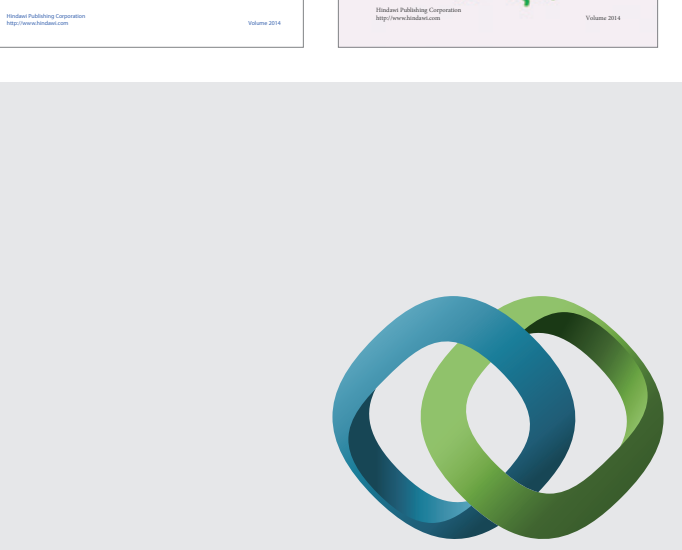

\section{Hindawi}

Submit your manuscripts at

http://www.hindawi.com
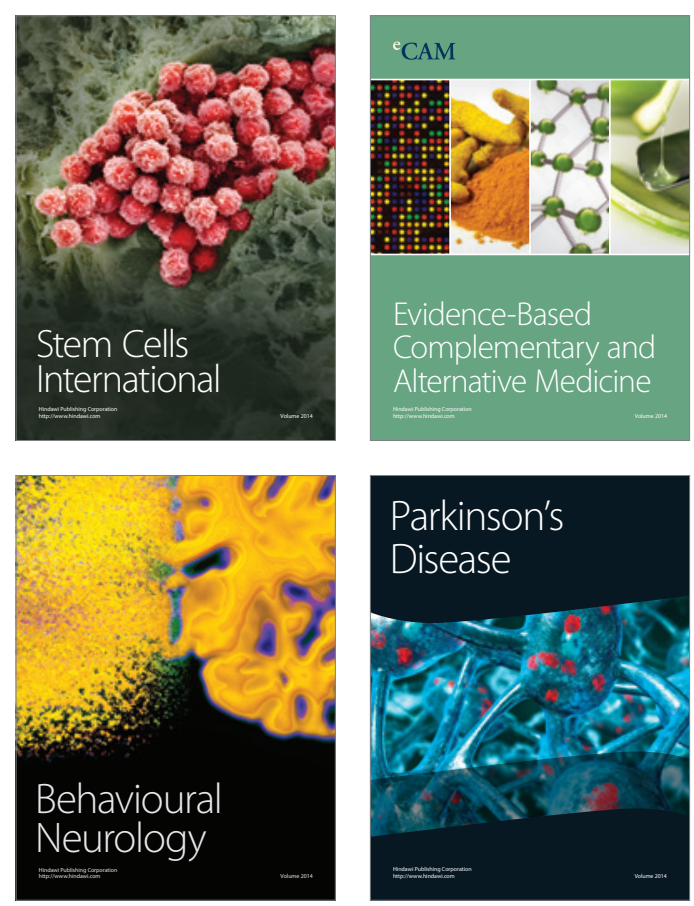

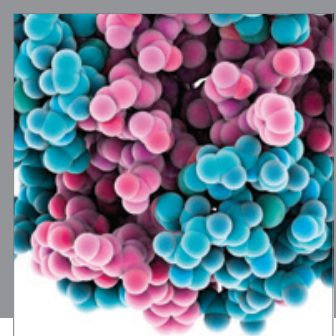

Journal of
Diabetes Research

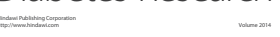

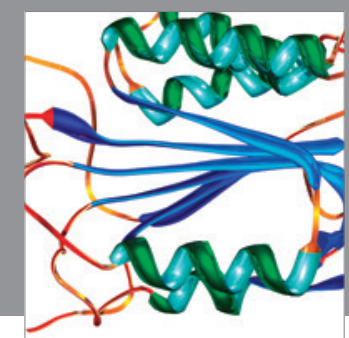

Disease Markers
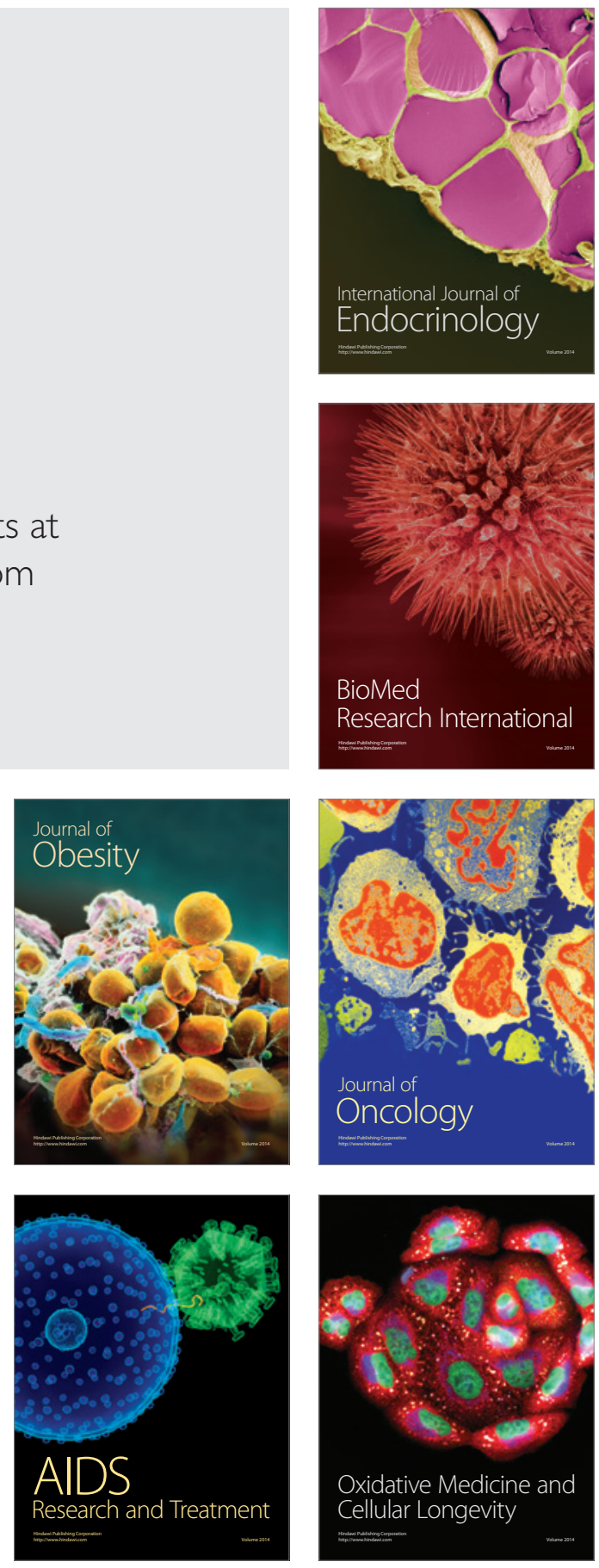